\title{
A novel PTC mutation in the BTB domain of KLHL7 gene in two patients with Bohring-Opitz syndrome-like features
}

\author{
Sara Cheraghi ${ }^{\mathrm{a}, \mathrm{d}}$, Sahar Moghbelinejad ${ }^{\mathrm{b}}$, Hossein Najmabadi ${ }^{\mathrm{c}}$, Kimia Kahrizi ${ }^{\mathrm{c}}$, Reza Najafipour ${ }^{\mathrm{b}, *}$ \\ ${ }^{a}$ Department of Molecular Medicine, Faculty of Medical Sciences, Qazvin University of Medical Sciences, Qazvin, Iran \\ ${ }^{\mathrm{b}}$ Cellular and Molecular Research Centre, Qazvin University of Medical Sciences, Qazvin, Iran \\ ${ }^{\mathrm{c}}$ Genetics Research Center, University of Social Welfare and Rehabilitation Sciences, Tehran, Iran \\ ${ }^{\mathrm{d}}$ Student Research Committee, University of Social Welfare and Rehabilitation Sciences, Tehran, Iran
}

\section{A R T I C L E I N F O}

\section{Keywords:}

Bohring-opitz-like syndrome

KLHL7

Whole exome sequencing

Iran

\begin{abstract}
A B S T R A C T
The bric-a-brac, tramtrack and broad complex (BTB) superfamily of conserved proteins are involved in ubiquitinproteasome system that contains the Kelch-like (KLHL) gene family. Kelch-like family member 7 (KLHL7), one of the KLHL gene family, consists of one BTB/POZ domain, one BACK domain and five or six Kelch motifs. Numerous variants in KLHL7 gene domains have been reported with Crisponi syndrome/cold-induced sweating syndrome type 1 (CS/CISS1)-like features and retinitis pigmentosa 42, and have recently been identified as causing Bohring-Opitz syndrome (BOS)-like features. We report two siblings with BOS-like phenotype with healthy parents and living in Qazvin province (Central Iran).

We performed whole-exome sequencing (WES) on the older patient and Sanger sequencing was carried out for validation of potential causative variants in the close family.

A novel homozygous frameshift mutation, p.(Phe83Leufs*3), was identified in the BTB domain of $K L H L 7$ that caused a premature translation-termination codon (PTC) in the two siblings with severe developmental delay, microcephaly, facial dysmorphism, peripheral retinal and optic disc atrophy and cardiac septal defects.

Our findings are in agreement with the clinical spectrum of KLHL7 mutations, which are associated with BOSlike features that reports for first time in our population.
\end{abstract}

\section{Introduction}

Kelch like family member 7 (KLHL7; OMIM\# 611119, 7p15.3) gene encodes a BTB (bric-a-brac, tramtrack and broad complex)-Kelch related protein with a BTB/POZ domain, a BACK domain and five or six Kelch repeats (Kanthi et al., 2018). KLHL7 is expressed at a high level in various human tissues such as neurons, lung, heart, testis and various cancer cells. In addition, KLHL7 expression has been demonstrated in the mouse embryonic nervous system (Bredholt et al., 2006; Diez-Roux et al., 2011). Proteins in the BTB family are involved in the ubiquitinproteasome system and in protein degradation. Protein degradation affects synaptic function, neuronal function and development, and has also been reported in various neurodegenerative disorders (Friedman et al., 2009). KLHL7 gene variants have been observed in several neurological diseases (Fig. 1), and dominant missense variants in a BACK domain of KLHL7 gene are responsible for retinitis pigmentosa 42 (RP42) (OMIM\# 612943, autosomal dominant) of Scandinavian origin (Friedman et al., 2009). A second associated disorder, Crisponi syndrome (CS)/cold-induced sweating syndrome type 1 (CISS1)-like (OMIM\# 617055, autosomal recessive) phenotype, was reported in four families of Turkish origin with autosomal recessive KLHL7 gene mutations (Angius et al., 2016). Recently, Bruel et al. reported a clinical spectrum of recessive mutations related to KLHL7. Autosomal recessive variants in the $K L H L 7$ gene were identified with acute clinical features associated with Bohring-Opitz syndrome (BOS) (Bruel et al., 2017). The authors studied six affected individuals from four families who had no mutations in ASXL1 (additional sex combs-like protein 1; MIM\# 612990, 20q11.21) gene, but various mutations identified in Kelch repeats of KLHL7 gene (Bruel et al., 2017). Also, very recently, a biallelic mutation in the BTB domain of KLHL7 has been identified in an affected individual with combined features of Bohring-Opitz-like and CS/CISS1-like phenotypes (Kanthi et al., 2018). Clinical features of both Bohring-Opitz-like and CS/CISS1-like phenotypes were also observed in two Guatemalan patients (Jeffries et al., 2018).

The ubiquitin-proteasome system is the significant mechanism for developmental and cell cycle regulation. The CULLIN-RING ubiquitin

\footnotetext{
* Corresponding author. Genetics, Cellular and Molecular Research Centre, Qazvin University of Medical Sciences, Shahid Bahonar Blvd, Qazvin, Iran.

E-mail address: rnajafipour@gmail.com (R. Najafipour).
} 